\title{
La gestione infermieristica dei cateteri venosi centrali nella emodialisi cronica
}

\author{
Giusy Lessio, Mara Canzi
}

\author{
U.O. di Emodialisi, A.O. S.Maria degli Angeli, Pordenone
}

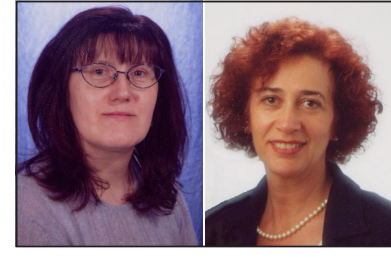

Giusy Lessio e Mara Canzi so vascolare che deve garantire elevati flussi ematici per determinare un'adeguata efficienza dialitica e una buona qualità di vita del paziente uremico. L'attuale configurazione della popolazione dialitica costituita in prevalenza da anziani con multipatologie, $\mathrm{DM}$, nefroangiosclerosi, vasculopatie obliteranti, ha fatto emergere il problema della gestione dei cateteri venosi centrali (CVC) che, considerata la difficoltà di allestire in tali soggetti un accesso vascolare interno (FAV), sono diventati spesso l'unica alternativa possibile. Nel nostro Centro i pazienti con accesso venoso centrale sono circa il $26 \%$ della popolazione dialitica; pertanto abbiamo sviluppato un protocollo di gestione e monitoraggio dei CVC per emodialisi.

Per la progettazione del protocollo ci siamo prefissati alcuni obiettivi:
1. migliorare le prestazioni assistenziali ottimizzando tempi e modi di svolgimento e l'uso di materiali;

2. garantire al paziente il massimo grado di sicurezza durante le diverse fasi operative;

3. ridurre i rischi (personali e ambientali) legati a manovre improprie;

4. proporre uno schema predeterminato di comportamento nelle diverse procedure;

5. fornire a tutti gli operatori le conoscenze necessarie per un'appropriata gestione dei CVC;

6. individuare e trattare adeguatamente le eventuali complicanze.

Ci siamo avvalsi dell'apporto della letteratura fondata sul razionale scientifico (EBM, EBN), delle indicazioni fornite dai CDC, della metodologia basata sulla VRQ, MCQ, TQM, dei riferimenti normativi specifici.

Il protocollo consta di alcune raccomandazioni:

1. limitare l'uso dei CVC per emodialisi al personale di dialisi ed esclusivamente per eseguire la dialisi; un uso alternativo dei
CVC viene consentito in caso di indisponibilità assoluta di altro accesso vascolare previa formazione specifica degli operatori;

2. adeguata antisepsi cutanea dell'emergenza preceduta da detersione, e successivo confezionamento di medicazione valutando per ogni paziente:

4 modalità di confezionamento (medicazione sterile in PU, in poliacrilato);

4 tempi di sostituzione;

4 materiale e disinfettanti adeguati;

3. prima e durante ogni utilizzo dei CVC eseguire lavaggio antisettico delle mani;

4. dopo ogni intervento sul paziente, lavarsi le mani;

5. non utilizzare agenti chimici, fisici, meccanici, potenzialmente dannosi per i CVC evitando anche trazioni e piegature dei dispositivi;

6. adottare tecniche rigorosamente asettiche per ogni procedura:

4 preparazione del circuito extracorporeo e delle apparecchiature di dialisi;

4 fase di avvio della seduta; 4 fase della reinfusione; 


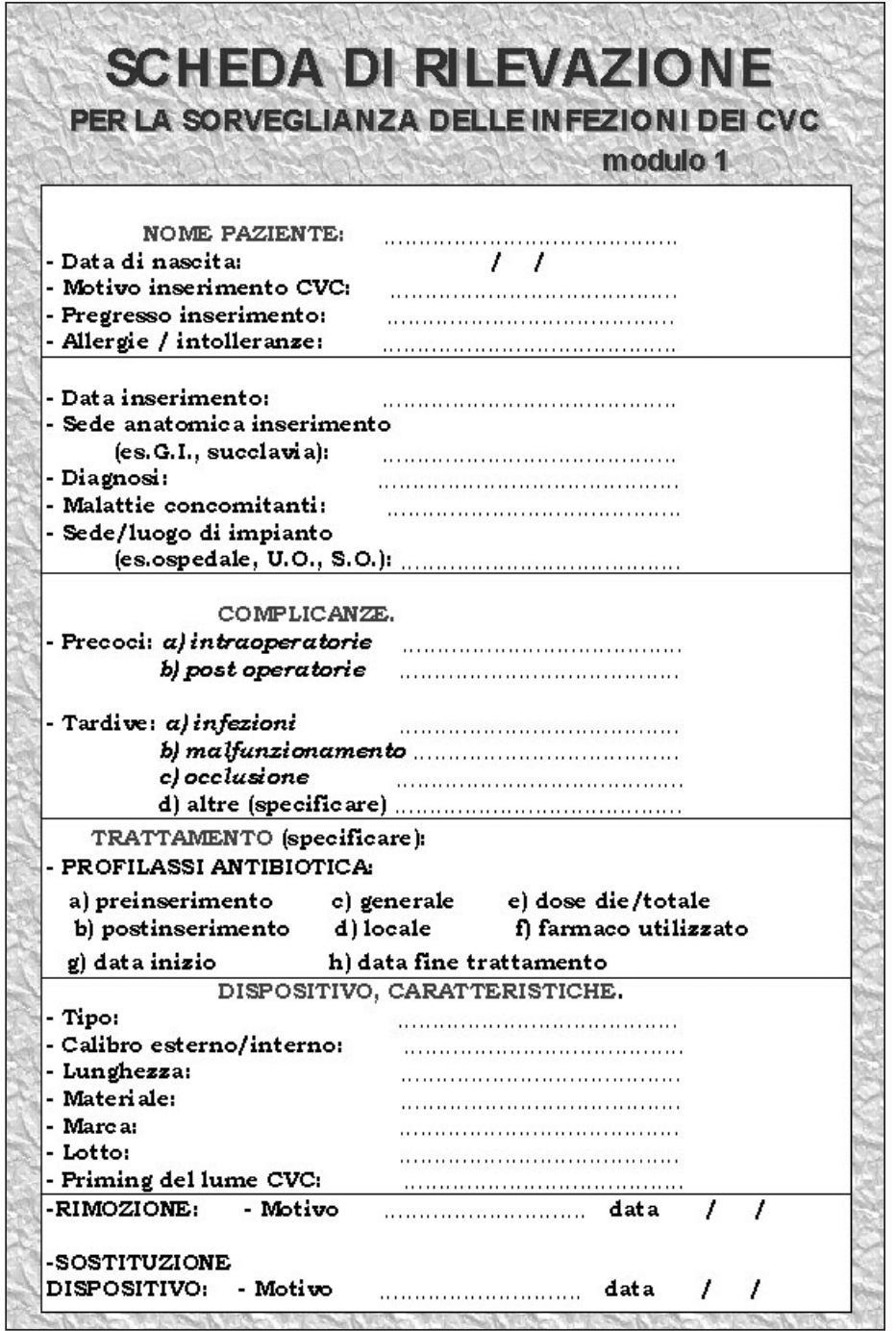

Fig. 1

4 preparazione e somministrazione della terapia;

4 eparinizzazione dei cateteri;

4 medicazione;

7. indossare i guanti prima di utilizzare i CVC, e utilizzarli in maniera adeguata (indossarli e rimuoverli, sostituirli, valutare quando utilizzare quelli sterili);

8. avvalersi dei dispositivi individuali di protezione:

4 camice, guanti, mascherina, occhiali, cuffia;

9. eseguire pulizia, detersione, disinfezione appropriata dei presidi, monitors, letti, ambienti ecc.

10. evitare la dispersione nell'ambiente di lavoro di fluidi e materiali del circuito extracorporeo; se ciò accadesse, detergere e disinfettare imfermieristica:

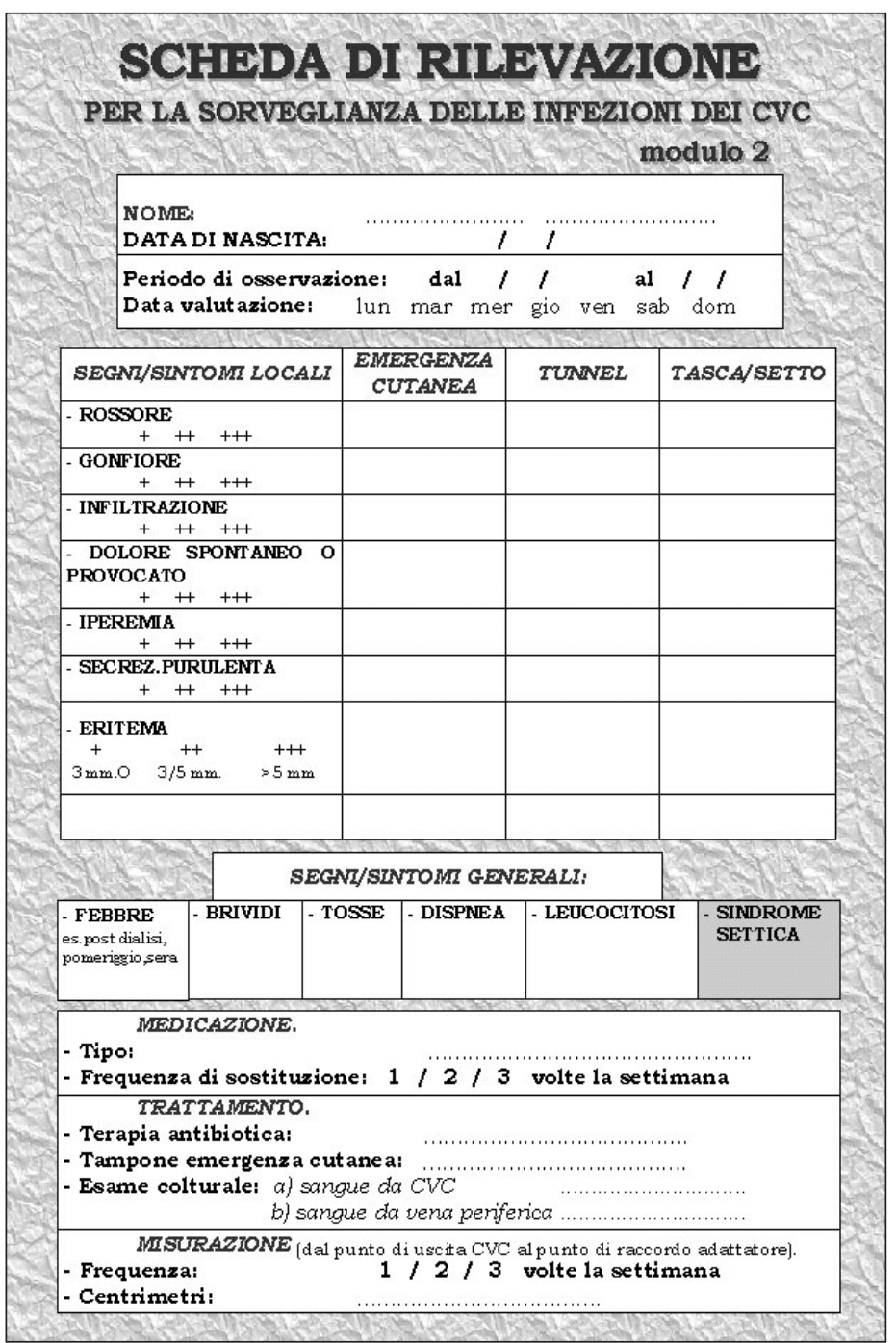

Fig. 2

mediatamente l'area contaminata;

11. seguire i protocolli prestabiliti per ogni singola procedura evitando manovre scorrette (aumentano il rischio di complicanze);

12. segnalare in apposita scheda in-

4 stato della cute della sede di medicazione;

4 condizione dell'emergenza cutanea, del tunnel sc;

4 stato dei cateteri, dei raccordi, dei tappi;

4 misurazione dei CVC (frequenza e discrepanze);

4 sostituzione della medicazione;

4 ispezione del sito (ora, giorno, motivazione);

4 comparsa di eventuali complicanze (segni e sintomi);
4 soluzioni e interventi effettuati. Abbiamo messo a punto uno strumento integrativo che consta di due schede di rilevazione per la sorveglianza delle complicanze (Figg. 1, 2). Tale strumento consente di individuare tempestivamente i primi segni o sintomi di complicanza e di avviare trattamenti adeguati ed efficaci per ridurre al minimo eventuali danni.

L'adozione del protocollo è indispensabile per garantire nel tempo un buon funzionamento dei CVC e una lunga durata dell'accesso vascolare e la sua precisa attuazione consente di prevenire e ridurre le complicanze che rappresentano dei fattori critici per la sopravvivenza dei pazienti in trattamento dialitico.

La revisione dei processi assistenziali 
permette di misurare gli effetti che le procedure e le cure hanno sullo stato di salute del paziente rendendo possibile l'obiettivo del miglioramento continuo della qualità delle cure.

Ma il presupposto fondamentale per lo sviluppo di uno stile comportamentale adeguato alla qualità dell'assistenza rimane la formazione continua degli operatori e una sistematica impostazione metodologica capace di integrare conoscenze e competenze specifiche; in grado cioè di prevenire gli eventi piuttosto che subirli.

giusyemaury@tin.it

\section{BIBLIOGRAFIA}

AA. Vari. Linee guida per la prevenzione delle infezioni associate a dispositivi intravascolari. Centers for disease control and prevention-HICPAC. GIIO 1997; n. 1 gennaio-marzo.

Stradoni R, Parrocchia S, Chiarelli C. Sorveglianza e prevenzione delle infezioni ospedaliere: "i casi indice" come eventi sentinella in emodialisi. G Ital Nefrolog 2000; 17: 11-5.

Mosconi G, Canova C, Bertuzzi V, Manna C, Colì L, Stefoni S. Rischio infettivo nelle manovre invasive in emodialisi. G Ital Nefrolog 2000; 17: 23-7.

Cagnoli L, Gattiani A. Controllare le manovre a rischio per prevenire le contaminazioni accidentali in emodialisi. G Ital Nefrolog 2000; 17: 28-36.

Emiliani G, Baldisserri P, Bartera C, Fantini E, Galassi P, Fusaroli M. Sistemi di protezione per la prevenzione delle infezioni associate ai dispositivi intravascolari. G Ital Nefrolog 2000; 17: 37-43.

Tarchini R, Botti PL, Talassi E, Lambertini D. Ottimizzare spazi e procedure in dialisi. G Ital Nefrolog 2000; 17: 44-9.

Milillo MG. Gli infermieri come strumento di prevenzione: competenza ed etica nel progetto globale di recupero della salute. Atti IV Congresso Nazionale ANIPIO, Genova, 1998; Novembre 4/7: 44-9.

Marchiaro G, Serra R. Strategie di sorveglianza dei patogeni emergenti. Atti IV Congresso Nazionale ANIPIO Genova, 1998; Novembre 4/7: 51-4.

Cavatorta F, Zollo A, Fiorini F. Il catetere venoso centrale in dialisi. Aspetti tecnici in Nefrologia. Direttore Andreucci VE, n. 11: 11-18, 56-67, 73-87. 